\section{STING/TYPE I INTERFERON PATHWAY ACTIVATION IN PATIENTS WITH PERNIOSIS DURING THE COVID-19 PANDEMIC}

${ }^{1}$ Jie An, ${ }^{2}$ Andrea Kalus, ${ }^{1}$ Sarah Chung, ${ }^{3}$ Brandon Seaton, ${ }^{2}$ Ata Moshiri, ${ }^{4}$ Daniel Lih, ${ }^{4}$ Ying Zheng, ${ }^{5}$ Hari Krishnamurthy, ${ }^{1}$ Xizhang Sun, ${ }^{1}$ Lena Tanaka, ${ }^{1,6}$ Keith B Elkon*. ${ }^{1}$ Division of Rheumatology, Department of Medicine, University of Washington, Seattle, WA; ${ }^{2}$ Division of Dermatology, Department of Medicine, University of Washington, Seattle, WA; ${ }^{3}$ Clinical Trials Pathology Lab, Fred Hutchinson Cancer Research Center, Seattle, WA; ${ }^{4}$ Department of Bioengineering, University of Washington, Seattle, WA; ${ }^{5}$ Vibrant America Clinical Lab, Vibrant America, San Carlos, CA 94070; ${ }^{6}$ Department of Immunology, University of Washington, Seattle, WA 98109

\subsection{6/lupus-2021-lupus21century.70}

Background During the COVID-19 pandemic, a high incidence of patients with perniosis was observed worldwide. Classically, perniosis is secondary to cold exposure, hemoproliferative and autoimmune diseases. Although the pathomechanism of perniosis is incompletely characterized, lupus pernio is associated with a Type I interferon (IFN-I) signature. Therefore we explored the role of IFN in patients with perniosis.

Methods Antibodies to SARS-COV-2 were tested by protein microarray. Expression of IFN-I, IFN stimulated genes (ISGs) and other inflammatory cytokines in peripheral blood were determined by qPCR. Inflammatory cytokine proteins in serum were quantified by Biolegend LegendPlex. Immunohistochemistry was used to detect expression of the IFN-induced protein, Myxovirus resistance protein A (MxA) in lesional skin. STING protein phosphorylation in CD14 monocytes was determined by flow cytometry. The effect of patient sera on microvesselson-a-chip was determined by Von Willebrand Factor (vWF) protein release to the vessel lumen. Statistical significance was determined by Student's t-test.

Results Between April-September 2020, 7 patients (3M;4F age 31-56) with pernio of the toes and/or fingers were studied (figure $1 \mathrm{~A}$ and $\mathrm{B}$ ). Two patients had previous Raynaud's phenomenon but none had prior or co-existent autoimmune disease. 1/5 patients tested was ANA+. 4/7 patients reported suspected COVID-19 symptoms prior to onset of perniosis. Antibodies to COVID antigens were negative in all patients.

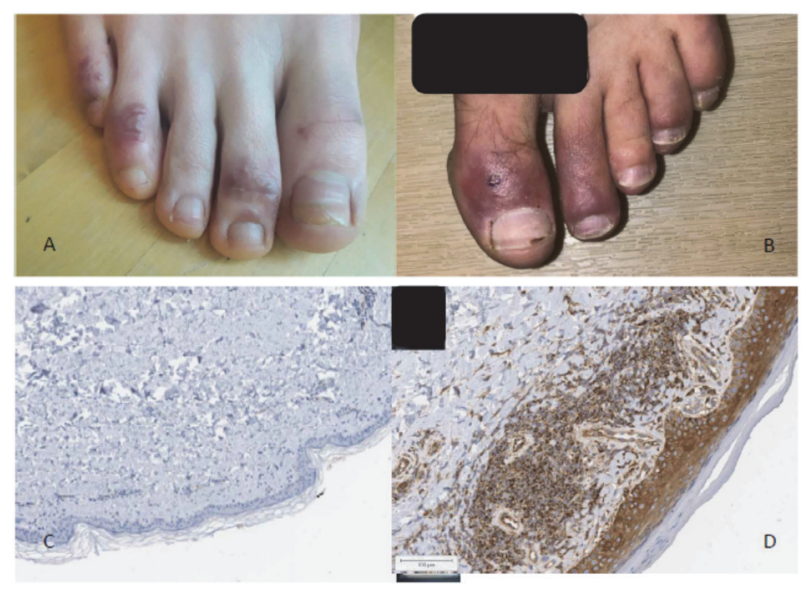

Abstract 1203 Figure 1 Clinical appearance of perniosis in study patients showing red and purple papules over several toes some with near blisters (A and B). MxA staining of skin from perniosis lesion (D) showing lymphocytic inflammation in the dermis with perivascular and periadenexal inflammation. There is prominent MxA staining in the epidermis, dermal inflammatory infiltrate and in the superficial endothelial cells indicating interferon activation in skin. This is compared to no MxA staining in normal skin (C).
Blood studies showed an increase in TNF gene expression in perniosis patients compared to healthy matched controls $(p=0.02)$. While there was a trend toward increased mRNA expression of IFNb $(p=0.07)$ and the ISG CXCL10 $(p=0.07)$, the results did not reach statistical significance. Phosphorylation of STING in CD14+ monocytes was higher in perniosis than healthy controls ( $n=3$ per group), with borderline statistical significance $(p=0.05)$. Lesional skin biopsies in perniosis $(n=2)$ showed striking expression of MxA in tissue (Fig1. C\&D). 4 patient sera induced high vWF release into the lumen on the microvessel 3D chip.

Conclusions The frequency of perniosis during the COVID pandemic, suggests a relationship between these two conditions although direct evidence of COVID-19 infection has been limited. We observed a trend toward higher IFN-b gene expression in PBMC as well as higher phospho-STING protein expression in CD14 monocytes and, most significantly, strong expression of $\mathrm{MxA}$ in skin. While the small number of patients preclude a definitive explanation, our data suggest that COVID associated perniosis is an interferonopathy. We propose that acute, transient COVID infection led to monocyte activation, IFN-I production and damage to the small vessels, likely aggravated by cold exposure.

\section{CHARACTERISTICS AND FACTORS ASSOCIATED WITH VACCINE HESITANCY IN A PREDOMINANTLY BLACK SYSTEMIC LUPUS ERYTHEMATOSUS COHORT}

S Sam Lim*, Charmayne Dunlop-Thomas, Gaobin Bao, Cristina Drenkard. Emory University, Department of Medicine, Division of Rheumatology, USA

\subsection{6/lupus-2021-lupus21century.71}

Background Communities of color are disproportionately impacted by COVID-19 and systemic lupus erythematosus (SLE). We explored factors associated with vaccine hesitancy (VH) in a SLE cohort.

Methods The Georgians Organized Against Lupus Cohort is a population-based cohort of adult, validated lupus patients in Atlanta, Georgia. Participants were surveyed by internet, mail, phone or in person beginning in April 2020 covering sociodemographics, medications, and psychosocial factors. An additional survey beginning January 2021 assessed vaccination perceptions. Frequency of responses through June 2021 were reported overall and by vaccine hesitancy status. Differences were evaluated using t-tests and P-values. Multivariate logistic regression analyses were conducted to explore factors associated with $\mathrm{VH}$.

Results Excluding those with severe reactions to previous vaccinations $(n=71)$, religious exemptions $(n=33)$, or diagnoses of primary cutaneous lupus $(\mathrm{n}=74), 598$ individuals (details in table 1) with SLE were predominantly female (93.3\%) and Black (78.4\%) with a mean age at survey of 51.4 years. Many lived in poverty $(25.5 \%)$, were unemployed $(35.6 \%)$, and had Medicare and/or Medicaid (51.6\%). Those endorsing $\mathrm{VH}$ $(42.1 \%)$ were younger, more often Black, less married, poorer, less educated, and had more Medicare and/or Medicaid compared to those who were vaccine receptive (57.9\%). They were significantly younger at diagnosis but with shorter disease duration. There were no differences in disease activity, damage, or medication use, except for more glucocorticoid use in the $\mathrm{VH}$ group. The $\mathrm{VH}$ group had less trust in the government, news, doctors, and lupus advocacy or support groups 